EPJ Web of Conferences 16, 01002 (2011)

DOI: $10.1051 /$ epjconf/20111601002

(C) Owned by the authors, published by EDP Sciences, 2011

\title{
The NETS Project: A NEtwork of transit surveys
}

\author{
I.-G. Jiang ${ }^{a}$ \\ Department of Physics and Institute of Astronomy, National Tsing-Hua University, \\ Hsin-Chu, Taiwan
}

\begin{abstract}
Motivated by the recent quick and successful development in the searching extrasolar planet (exoplanet) by transit events, I started an instrumental project, the NETS Project, in 2006. In this paper, the background, goal, our design, the progress, and the current status will be reviewed. Although our research grant and manpower are limited, we are approaching to the stage to build up a station at an astronomical observatory.
\end{abstract}

\section{INTRODUCTION}

The discovery of about 400 planets orbiting other stars, i.e. exoplanets, has opened a new field of astronomy. It is particular interesting and important due to the potential to address fundamental questions about our Solar system and also the theories of planetary formation.

There are many interesting and exciting results about exoplanets these days. For example, Galland et al. (2005) found a exoplanet around the F6V star HD33564 showing a strong infrared excess. Baraffe et al. (2005) studied the origins of hot-Jupiters and hot-Neptunes. The formation of hot-Jupiter is particularly interesting due to the discovery of OGLE transiting exoplanets. The existence of exoplanets can explain the asymmetric signatures of debris disc (Ozernoy et al. 2000). The Beta Pictoris' warp debris disc could be explained by an exoplanet with an inclined orbit.

Moreover, there are many dynamical studies for the orbital evolution of exoplanets. (Please see Laughlin \& Adams 1999, Rivera \& Lissauer 2000, Jiang \& Ip 2001, Ji et al. 2002.) The richness of the dynamical work is mainly because these exoplanets exhibit many interesting and surprising features: high-eccentricity orbits, extremely short-period orbits and mean-motion resonances etc.

My group also has contributed on this topic. For example, Jiang \& Ip (2001) showed that the orbital configuration of the planetary system of upsilon Andromedae might be caused by the disc interaction initially. Yeh \& Jiang (2001) studied the orbital migration of scattered planets. Jiang \& Yeh (2004) did some analysis on the chaotic orbits for dynamical systems of star-planet-belt interaction and Jiang et al. (2003) studied the tidal interactions between the host stars and close-in planets. In addition, Jiang et al. (2006) used the statistical methods to do the data analysis for the distributions of exoplanets in their parameter spaces.

The transit searches so far yields about 60 planets in total. This is encouraging because, for a transiting planet, the orbital inclination can be estimated from the light-curve, and thus the actual planetary mass can be measured. Measurements of radial velocity variations in the host star yield only a minimum mass.

ae-mail: jiang@phys.nthu.edu.tw

This is an Open Access article distributed under the terms of the Creative Commons Attribution-Noncommercial License 3.0, which permits unrestricted use, distribution, and reproduction in any noncommercial medium, provided the original work is properly cited. 
There are many on-going projects of planetary transit surveys. For example, PASS, Vulcan-S, HAT1, WASP0, ASAS-3, PPS, PSST, STARE, SuperWASP, PISCES, ASP, OGLE-III, EXPLORE etc. The apertures range from $3.6 \mathrm{~cm}$ to $360 \mathrm{~cm}$. The field of view is from 0.57 degree to 108 degree. Some of these projects are special-target transit surveys. For example, the transit surveys on the globular cluster, open cluster or the Galactic plane. Some of them are wide-field surveys. In general, the telescopes with smaller fields of view (with larger apertures) do the surveys on the chosen particular targets, the telescopes with larger fields of view (with smaller apertures) can do sky surveys. Thus, one has to make a decision from either deep or wide. The wide field surveys can only study brighter stars in the Solar neighborhood and therefore not necessary to worry about the geometry of the Milky Way.

Among these detected transiting exoplanets, at the early stage, most of them are detected by the $1.3 \mathrm{~m}$ OGLE telescope. It is exciting that the $10-\mathrm{cm}$ telescope of the Trans-Atlantic Exoplanet Survey (TrES) network detected a remarkable transit event and confirmed the discovery of an exoplanet: TrES1 in 2004. Moreover, they have a another discovery, TrES-2, in 2006. The field of view of the TrES telescope is 6 degree. It is CCD-based system with spatial resolution of about $11 \mathrm{arcsec} / \mathrm{pixel}$.

Motivated by the above development, I decided to construct a set of low cost instruments to do sky surveys for transit events. I have the research grant supported by National Science Council in Taiwan, which is just enough to start a feasibility study from 2006.

\section{THE GOAL}

Among these on-going projects of transit surveys, there are two different types of projects: (i) the special target surveys by telescopes with larger aperture, (ii) the wide-field surveys by telescopes with smaller aperture. It is difficult to predict which kind would be more successful because one can find that the total numbers of stars they can monitor are compatible and the detection probability is proportional to the total number of monitored stars. It is a good news that the low-cost systems of small aperture can compete with the meter-class telescopes in terms of the transit surveys.

One of the problems for these on-going projects is that the number of stars they can monitor is very small, comparing with the total number of stars on the sky within their limiting magnitude. It is indeed difficult to cover the entire sky. For example, assuming 2 months per field with a field of view 10 degree by 10 degree, it would take 60 years for a single 10-degree CCD camera to survey the entire sky.

Let us estimate the number of stars. The star's number density is about $0.3 \mathrm{pc}^{-3}$ in the Milky Way's disk. The potential total number of monitoring stars is about $0.3 \times(4 / 3) \pi \times(200) \times(200) \times(200)$, which is about 10,000,000 (for $7 \mathrm{~cm}$ telescope). For $12 \mathrm{~cm}$ telescope, it can observe stars up to about 500 pc, which is still within the thickness of the Milky Way's disc. The potential total number of monitoring stars is about $0.3 \times(4 / 3) \pi \times(500) \times(500) \times(500)$, which is about $160,000,000$. Please note that this is just a quick and simple estimation. More specific models are needed for the details.

However, the typical number of monitoring star is only about a few tens thousand for all the on-going projects. How do we solve the above problems? How do we increase the number dramatically?

For our long-term goal, we propose to have a special array of small aperture telescopes. At each observational site, there would be an array of non-tracking systems, which can scan the entire sky quickly. These telescopes would point to different declination. While the Earth rotates, they scan stars toward different right ascension.

The reason to have the non-tracking systems is to scan the entire sky quickly. If the field of view is 30 degree, we will need about 4 such systems. If the field of view is 7 degree, we will need 10 or 20 such systems.

On the other hand,if the field of view is $30 \mathrm{deg}$., we only can monitor the stars for about 2 hours by non-tracking systems. If the field of view is 7 degree, we only can monitor the stars for about 0.5 hour by non-tracking systems. We therefore might not have the complete light-curves. 
In order to follow up and complete the light curve, we will set up some collaboration with a few observatories. In this way, we can increase the total number of stars dramatically on one hand, and on the other hand, we can still have nice complete transit light curves.

\section{THE ADVANTAGES}

In this section, we discuss the advantages of an array of non-tracking systems.

(1) The Number of Scanned Stars:

As we estimated in the previous section, the potential number of target stars is huge but the usual tracking system would only scan a limited number of stars. Our system will monitor much more stars.

(2) A Wider Range of Magnitudes:

When one wishes to observe fainter stars, one needs to use longer exposure time. However, the brighter stars in the same image would get saturated if its brightness is already out of the dynamical range of CCD. This would make it difficult to have useful data for both brighter and fainter stars. For our non-tracking system, the photon comes to different pixels when the stars move. It would not get saturated as easy as for the tracking system.

(3) A Low Cost:

The major cost would be the lens and CCD Camera. Therefore, it is extremely easy to expand to be an array or to have multiple systems. The number of monitoring stars is proportional to the number of systems.

(4) Simple and Robust:

The structure of our system is very simple. It can be used for a long time. Therefore, it is very good for a long-term sky survey.

(5) Easier Image Registration:

For tracking systems, usually the stars' photons would not come to exactly the same pixels of CCD. One needs to re-register images to determine the possible stellar energy flux variations. For our system, the stars' photons come to the same pixels at the same sidereal time when the stars have no proper motion.

(6) Easier Air-Mass Correction:

For our system, the stars come into the field of view at the similar viewing angles. The air-mass correction factors are similar for this situation. Moreover, the sky background subtraction is also easier for the same reason.

\section{THE DISADVANTAGE}

Because we do not track stars, we only monitor each star for 0.5 to 2 hours every night. This is shorter than the one for the tracking systems because they can follow the stars whole night. The probability to miss some transit events is higher for us.

Nevertheless, this issue would not make the planet detection rate less than the rate of tracking systems. Because for short period planets, the transit events would repeat again and again. We will finally detect them anyway. For long period planets, our systems are more robust and can do very longterm surveys, we might have more chances to detect them.

\section{THE PROGRESS}

From what we have described above. The long-term goal appears to be an ambitious but low-cost project. However, both the instrumentation and data analysis of our special array of telescopes are different from the traditional tracking systems. We therefore start from a feasibility study. Here the progress is 


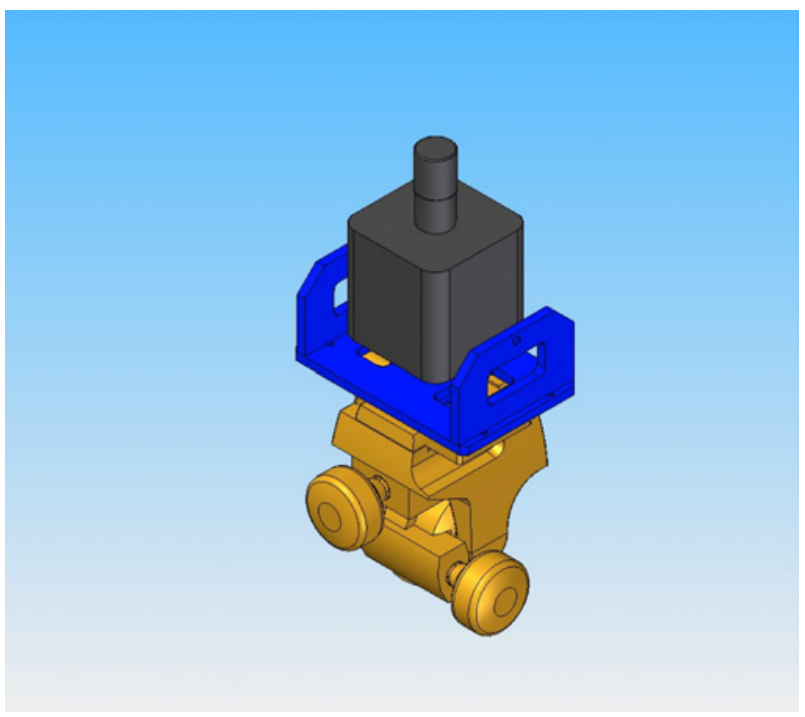

Figure 1. The CCD-lens system.

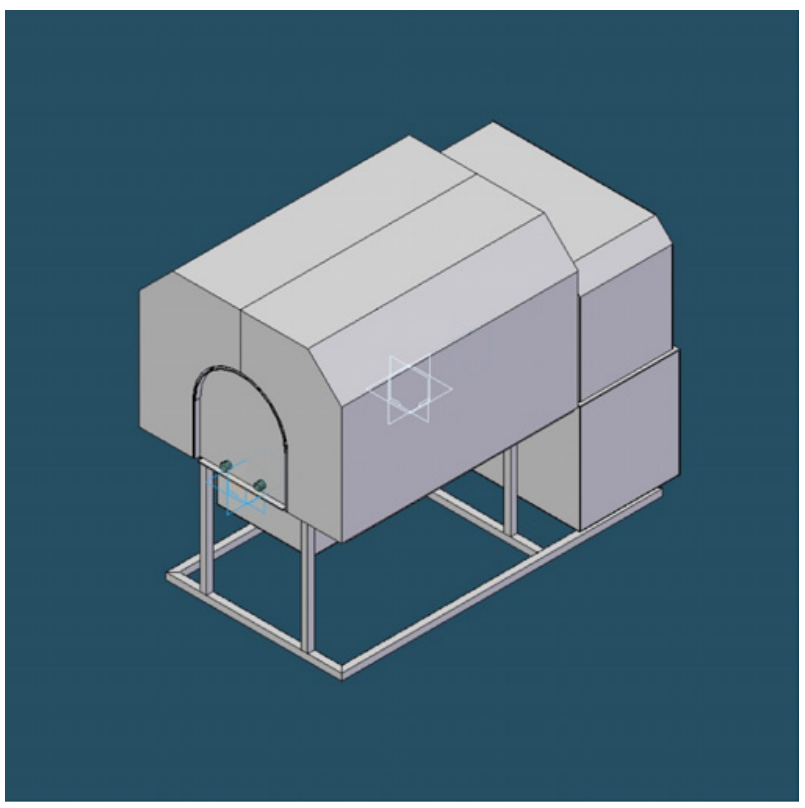

Figure 2. The closed enclosure.

summarized. In Oct. 2006, a prototype CCD-lens system was constructed. Fig. 1 shows the CCD-lens system with a platform. In Jan. 2007, It took some testing data at Lulin Observatory. In Feb. 2007, we started the code development. Then, we began to plan the enclosure in March 2007. Fig. 2 and 3 show our original design for the enclosure. In July 2007, our weather station arrived. In Nov. 2007, a new computer arrived. In Dec. 2007, the enclosure is constructed and completed. In March 2008, we completed the code which does the automatic control for the enclosure. In June 2008, a CCD is installed. Finally, in Nov. 2008, at our campus, Tsing-Hua Station was working and taking images 
Research, Science and Technology of Brown Dwarfs and Exoplanets

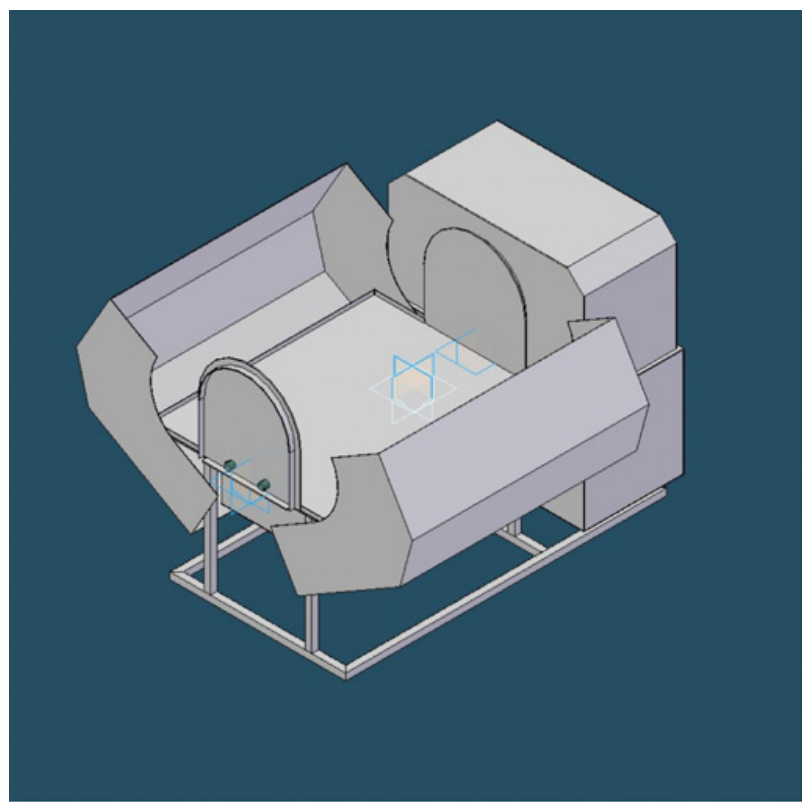

Figure 3. The opened enclosure.

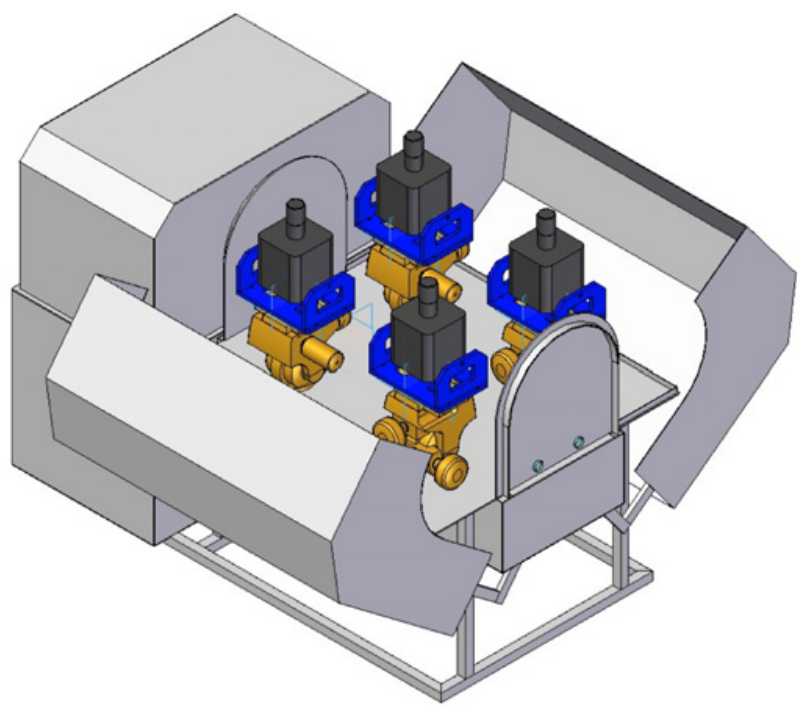

Figure 4. The enclosure with installed CCD-lens systems.

regularly. It works in a fully automatic observational mode and has accumulated more than 100 GB data of images. These images are very helpful for the development of the data-analysis software. Another CCD is installed in 2009, so we now have two CCDs working in the enclosure. Fig. 4 shows the picture when an array of four CCDs is completed in the future.

Once the time interval of observations is set, the computer checks if the weather condition is satisfied and electric power is normal, then the enclosure could open on time. The CCD camera takes images whenever the enclosure is open and stops when it is closed. The enclosure would close whenever the 


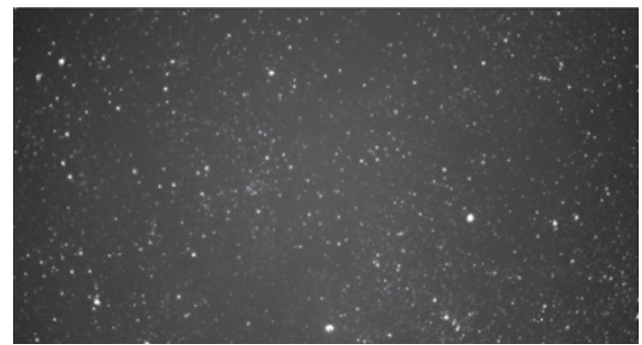

Figure 5. One of our typical images.

weather condition is not satisfied or the electric power is off. Once the time is up, the enclosure is also closed.

One of our typical images is shown in Fig. 5. At our campus, the ADU value of the sky background is about 3000. We found that many stars have ADU values about 40000 to 50000 Thus, for these stars, the signal to noise ratio is more than 10 . The flux variation of these stars is about several percents.

\section{CONCLUDING REMARKS}

Our project to build a network of transit surveys (NETS Project) is going well. We are now at the status to develop the data pipeline and search an oversea location to build a station which can obtain scientific data. We acknowledge the financial support from National Science Council, Taiwan, and hope the continuous support to help us to move ahead this low-cost but ambitious project to be on the stage of this field.

\section{References}

[1] Baraffe, I., et al. 2005, A\&A, 436, L47

[2] Galland, F., et al. 2005, A\&A, 444, L21

[3] Ji, J., Li, G., Liu, L. 2002, ApJ, 572, 1041

[4] Jiang, I.-G., Ip, W.-H. 2001, A\&A, 367, 943

[5] Jiang, I.-G., Ip, W.-H., Yeh, L.-C. 2003, ApJ, 582, 449

[6] Jiang, I.-G., Yeh, L.-C. 2004, AJ, 128, 923

[7] Jiang, I.-G., Yeh, L.-C., Hung, W.-L., Yang, M.-S. 2006, MNRAS, 370, 1379

[8] Laughlin, G., Adams, F. C. 1999, ApJ, 526, 881

[9] Ozernoy, L. M., et al. 2000, ApJ, 537, L147

[10] Rivera, E. J., Lissauer, J. J. 2000, ApJ, 530, 454

[11] Yeh, L.-C., Jiang, I.-G. 2001, ApJ, 561, 364 